\title{
AVALIAÇÃO DA DOR LOMBAR E DO CONTROLE MOTOR DA REGIÃO LOMBOPÉLVICA EM LUTADORES DE JIU-JITSU
}

\section{EVALUATION OF PAIN AND MOTOR CONTROL OF THE LUMBOPELVIC REGION IN JIU-JITSU}

\author{
Jéssica Emanoela Galdino da Costa ${ }^{1}$, Denis Vieira Lima ${ }^{2}$, Lúcio Frigo ${ }^{3}$ \\ ${ }^{1}$ Graduanda do Curso de Fisioterapia da Universidade Mogi das Cruzes - SP. E-mail: \\ emanuela.galdino@gmail.com \\ ${ }^{2}$ Fisioterapeuta, Mestre em Ciências da Saúde, Professor de Graduação da Universidade Mogi \\ das Cruzes - SP. E-mail: denis.lima255@gmail.com \\ ${ }^{3}$ Doutor em Ciências (Biologia Celular e Tecidual), Professor Titular de Pós-Graduação da \\ Universidade Cruzeiro do Sul. E-mail: luciofrigo@uol.com.br
}

Data de recebimento: $22 / 10 / 2013$

Data da aprovação: 11/12/2013

\begin{abstract}
RESUMO
Jiu-jitsu significa arte suave e tem como objetivo derrotar o adversário por meio de projeções, estrangulamentos, torções e/ou imobilizações. A dor lombar pode afetar o controle motor, tanto por inibição do motoneurônio e da alteração do impulso proprioceptivo, como também por influência direta do centro motor, afetando o planejamento motor. O objetivo deste trabalho é analisar o controle motor da região lombopélvica em lutadores, pelo método de biofeedback de pressão, e correlacioná-lo com a dor lombar. É um estudo observacional, descritivo e transversal, com intuito de inferir possíveis relações entre a dor lombar e o défice do controle motor dos músculos lombopélvicos. Para a avaliação foram realizados três testes, constituídos por movimentos com os membros inferiores: flexão, extensão e abdução/rotação lateral de quadril, e flexão de joelho. Resultados: entre os 80 lutadores, 32 relataram lombalgia. Com referência aos testes, apenas 13 conseguiram manter o controle motor no teste BKFO do lado direito, e do lado esquerdo foram 16. No teste KLAT, do lado direito, nas angulações de $30^{\circ}, 60^{\circ}$ e $90^{\circ}$, conseguiram manter o controle respectivamente $28,25 \mathrm{e}$ 12 lutadores; já do lado esquerdo, nas angulações $30^{\circ}, 60^{\circ}$ e $90^{\circ}$, conseguiram manter o controle respectivamente 29,22 e 14 lutadores. No teste de extensão do quadril do lado direito, apenas 6 mantiveram o controle, e do lado esquerdo, apenas 8. Conclusão: a amostra analisada não apresentou controle motor lombopélvico pelo método de avaliação. Os testes estatísticos não apresentaram associação entre défice do controle motor lombopélvico e a dor lombar.
\end{abstract}

Palavras-chave: Jiu-jitsu. Dor lombar. Controle motor.

\begin{abstract}
Jiu-Jitsu means gentle art, and aims to defeat the opponent by means of projections, bottlenecks ,twists and / or assets. Low back pain (LBP) can affect motor control, both the inhibition of motoneuron and modified proprioceptive impulse thus changing the motor control, but also affects motor planning, directly influencing the motor center. Objective: to analyze the motor control of the lumbopelvic region into fighters by pressure biofeedback, and correlate the deficit of motor control LBP. Methodology: this is an observational, descriptive, cross-sectional view to infer possible relationships between lower back pain and motor control deficits lumbopelvic. For evaluation was conducted three tests that consist of movements with the lower limbs, flexion, extension
\end{abstract}


and abduction/lateral rotation of hip and knee flexion. Results: among the 80 fighters, 32 reported LBP. In tests, only 13 managed to keep the motor control test BKFO right and left side were 16 . Test KLAT the right angles in $30^{\circ}, 60^{\circ}$ and $90^{\circ}$ were able to maintain control respectively 28,25 and 12 fighters, since the left side in the angles $30^{\circ}, 60^{\circ}$ and $90^{\circ}$ were able to maintain control respectively 29,22 and 14 fighters. The test hip extension on the right side only 6 remained in control and the left side only 8 . Conclusion: The sample showed no motor control lumbopelvic by valuation method. Statistical tests showed no association between childhood motor control lumbopelvic and LBP.

Keywords: Jiu-jitsu. Low back pain. Motor control.

\section{Introdução}

O jiu-jitsu, como uma arte de defesa pessoal, surgiu há 2.500 anos. Naquela época, a prática era exercida apenas por monges budistas que habitavam uma região situada entre a Índia e a China. Esses monges tinham biótipo físico considerado como fraco e, por causa disto, desenvolveram uma técnica de defesa pessoal que lhes permitia se desviarem de ataques provenientes de armas brancas utilizadas pelos seus agressores; por vezes, a utilização dessa técnica possibilitava que eles ficassem em melhor situação que seus oponentes (GURGE, 2003).

A partir do final do século XIX, alguns mestres de jiu-jitsu migraram do Japão para outros continentes, difundindo assim o ensino de sua arte marcial. Esai Maeda Koma, conhecido como Conde Koma, foi um deles. Depois de viajar por diversos países, Koma chegou ao Brasil em 1915 e se fixou em Belém do Pará, onde conheceu Gastão Gracie. Gastão era pai de oito filhos, entre eles Carlos Gracie, que foi apresentado à luta com 15 anos de idade e, desde então, passou a transmitir os seus conhecimentos aos irmãos, adequando e aperfeiçoando a técnica (Confederação Brasileira de Jiu-jitsu).

Ao modificar as regras internacionais do jiu-jitsu japonês em relação às lutas que ele e os irmãos realizavam, Carlos Gracie iniciou o primeiro caso de mudança de nacionalidade na história esportiva mundial. Anos depois, a arte marcial japonesa passou a ser denominada de jiu-jitsu brasileiro, sendo exportada para o mundo todo (Confederação Brasileira de Jiu-jitsu).

A palavra jiu-jitsu significa arte suave e, quando praticado por atletas no âmbito profissional, tem como objetivo derrotar o adversário por meio de projeções, estrangulamentos, torções e/ou imobilizações, que são resultado de forças tensivas sobre as articulações. Tais gestos exigem diferentes capacidades do sistema musculoesquelético, buscando a desistência do adversário pelas técnicas especificas que se baseiam em posições de alavanca biomecânica (SOUZA et al., 2011; VECCHIO et al.,2007).

O praticante adulto utiliza o sistema de graduação por diferenciação de cor, composta por cinco estágios, conhecidos como faixas. Inicia-se pela faixa branca, a qual não necessita de tempo e idade mínima para alcançar o segundo estágio, a faixa azul. Com dois anos de treinamento, o lutador pode passar para a terceira graduação, composta pela faixa roxa, e, apresentando mais um ano e meio de treinamento, poderá atingir a faixa marrom. A última etapa, a faixa preta, que dá ao portador o título de mestre instrutor, poderá ser almejada após um ano da aquisição da faixa marrom. O tempo necessário para alcançar a mudança de graduação pode variar conforme a aquisição dos conhecimentos, técnicas e habilidades necessárias exigidas em cada faixa (Confederação Brasileira de Jiu-jitsu).

Em um estudo comparativo realizado com 94 praticantes de artes marciais, entre elas o jiu-jitsu e o karatê shotokan, as lesões mais comuns apresentadas pelos lutadores de jiu-jitsu ocorreram nas seguintes regiões: joelho $(16,3 \%)$, ombros $(14,4 \%)$ e orelhas (13,3\%) (SOUZA et al., 2011).

Durante a prática da luta olímpica, é exigido dos atletas uma grande força e potência dos músculos flexores do quadril. Além disso, há também a frequente realização do mesmo movimento do quadril, a fim de abaixar o centro de massa e aumentar a estabilidade durante as manobras defensivas. Esses movimentos têm sido descritos como prejudiciais à coluna vertebral devido à sobrecarga imposta sobre 
suas unidades funcionais, principalmente durante os rápidos movimentos de flexão de tronco. (DEZAN et al.,2004). O excesso de compressão resulta no aumento da carga mecânica na região interna do disco intervertebral, sendo considerado um fator de risco para os processos degenerativos, além de gerar dor na região lombar da coluna vertebral (HODGES et al., 2003; HODGES, 2011).

A estabilidade da coluna acontece por causa da interação de três sistemas: passivo, ativo e neural. O sistema passivo é composto pelas vértebras, discos intervertebrais, articulações e ligamentos, que fornecem a maior proporção de estabilidade pela limitação passiva ocorrida no final do movimento. O segundo é o sistema ativo, constituído pelos músculos e tendões, que favorecem o suporte e rigidez intervertebral para sustentar forças exercidas durante as atividades de vida diária. A contração máxima da musculatura é exigida em uma pequena proporção, cerca de $10 \%$ de coativação muscular é necessário à manutenção da estabilidade da coluna vertebral (PANJABI, 1992).

O último componente estabilizador a ser considerado é o sistema neural, composto pelo sistema nervoso central e periférico. Esses sistemas são responsáveis pela coordenação da atividade muscular em resposta às forças de estiramento e tensionais, que fornecem a estabilidade dinâmica. $\mathrm{O}$ sistema nervoso deverá interagir como o sistema ativo para que ocorram contrações musculares específicas em dado período de tempo, objetivando o correto movimento e consequentemente a proteção contra lesões (PANJABI, 1992).

Quando há um défice no desempenho muscular de grupos específicos, como o músculo transverso do abdome e os multífidos da região lombar, poderá ser desencadeada uma instabilidade biomecânica da coluna vertebral, além de se propiciar o aparecimento de processos álgicos e uma predisposição à fadiga muscular (BARBOSA et al., 2005).

Em um estudo foi utilizada a eletromiografia de superfície para analisar a atividade dos músculos abdominais e multífidos lombares durante os movimentos dos membros inferiores. Concluiu-se que a contração da musculatura do tronco ocorre em um período anterior ao início do movimento dos membros inferiores, independente da direção do movimento. Com isso, verificou-se que o retardo da resposta muscular do tronco apresenta um grande potencial para provocar uma instabilidade lombopélvica, sendo um fator de risco para as lombalgias crônicas (BARBOSA et al., 2005).

A dor lombar pode afetar o controle motor dos músculos do tronco de forma multifatorial, tanto com a inibição do motoneurônio e também da alteração do impulso proprioceptivo, alterando assim o controle motor. Porém, a proposta mais aceita é de que a dor lombar afeta o planejamento motor, influenciando diretamente no centro motor, e também pela alteração do sistema sensorial (HODGES et al., 2003; HODGES, 2011).

Há modelos sugestivos da interação entre os défices de controle motor e a alteração da propriocepção articular, desenvolvendo microtraumas, processos álgicos e riscos de lesões musculoesqueléticas (HODGES et al., 2003).

O controle motor da coluna vertebral e a sua estabilidade são dependentes da contribuição integrada entre os elementos passivos e os músculos adjacentes. No entanto, é de suma importância a acuidade do sistema neural, que detecta o estado de estabilidade e planeja a atividade muscular do tronco com antecedência às perturbações da coluna vertebral (HODGES et al., 2003).

O dispositivo de biofeedback de pressão foi originalmente desenvolvido para avaliar a capacidade dos músculos abdominais em estabilizar ativamente a região lombar da coluna vertebral e também avaliar o controle motor da região lombopélvica. Ele foi utilizado em diversos estudos como método de avaliação da estabilização lombar e considerado um instrumento confiável e válido para avaliar a função dos músculos abdominais profundos (PHROMPAET et al., 2011; HERRINGTON et al., 2005).

O instrumento de biofeedback de pressão é constituído por uma almofada inflável ligado a um medidor de pressão (manômetro) e um dispositivo de insuflação. A correta utilização deste instrumento segue as seguintes recomendações:

- Indivíduo deitado em decúbito dorsal ou ventral, com a unidade de biofeedback insuflada e posicionada entre a lordose lombar ou entre as espinhas ilíacas anterossuperior;

- O indivíduo é orientado a manter uma pressão constante registrada no manômetro, independente dos movimentos dos membros inferiores sob cargas externas. 
Mudanças da pressão durante o movimento do quadril refletem uma incapacidade de manter a contração isométrica dos músculos abdominais, o que resulta em uma instabilidade da coluna lombar e sugestiva falta de controle motor da região lombopélvica (PHROMPAET et al., 2011; ROUSSEL et al., 2009).

Este trabalho é justificado pela alta ocorrência dos processos álgicos da região lombar da coluna vertebral em lutadores (DEZAN et al., 2004) e a possível relação existente com a falta de controle lombar (HODGES et al., 2003). Outra justificativa seria o crescente número de praticantes e a escassez de pesquisas científicas com enfoque nessa população.

\section{Objetivo}

Analisar o controle motor da região lombopélvica em lutadores de jiu-jitsu por meio do instrumento de avaliação de biofeedback de pressão, e correlacionar o défice do controle motor com a sintomatologia de dor na região lombar da coluna vertebral.

\section{Materiais e métodos}

Este estudo foi aprovado pelo Comitê de Ética e Pesquisa da Universidade Mogi das Cruzes- UMC/ SP, parecer $n^{\circ}: 292.998$, datado de 28/05/2013. Está de acordo com suas atribuições definidas na Resolução CNS 196/96, que define as diretrizes e normas regulamentadoras de pesquisas envolvendo seres humanos.

Trata-se de um estudo observacional, descritivo de delineamento transversal, com intuito de inferir possíveis relações entre a dor lombar e o défice do controle motor lombopélvico em lutadores de jiu-jitsu.

\section{Amostra}

Para compor a amostra foi analisada a população de lutadores de jiu-jitsu da academia Power Lottus Team - Aricanduva, composta por 100 lutadores. O cálculo amostral determinou uma amostra de 80 lutadores do gênero masculino, com idade entre 18 a 42 anos, praticantes da arte marcial há pelo menos nove meses; com frequência de treino entre duas a quatro vezes por semana, com ou sem sintomatologia de dor na região lombar da coluna vertebral.

A presença de dor foi caracterizada pela Escala visual analógica (EVA) com escala maior ou igual a cinco pontos, do máximo de dez pontos.

\section{Critérios de exclusão}

Foram excluídos da pesquisa os indivíduos que apresentaram: histórico clínico de cirurgia abdominal ou pélvica num período inferior a 12 meses; indivíduos que estejam realizando tratamento analgésico (medicamentoso, ou outros); indivíduos que se apresentaram em período de convalescência de doenças inflamatórias ou infecciosas; indivíduos que sofreram trauma ortopédico na região abdominal, pélvica ou lombar, na última semana antecedendo a coleta de dados, além do estipulado na amostragem; e os indivíduos participantes do estudo piloto.

\section{Material}

O controle motor foi avaliado pelo biofeedback de pressão (marca Chattanooga, modelo Stabilizer ${ }^{\circledR}$ ), constituído de uma almofada inflável e um manômetro analógico para visualização da variação de pressão, além de um dispositivo para a insuflação.

O equipamento apresenta um valor basal de 40 mmHg, e mudanças dessa pressão indicam movimentação da região lombopélvica, determinando o défice do controle motor. Alteração de até $2 \mathrm{mmHg}$ (38 a $42 \mathrm{mmHg}$ ) são desconsideradas, pois referem-se aos movimentos de inspiração e expiração dos voluntários (HERRINGTON et al., 2005).

Foi utilizado também um goniômetro universal (marca Carci) para mensurar a angulação adequada de cada movimento proposto pelos testes.

\section{Procedimentos}

Todos os voluntários foram obrigados a ler a carta de informação e o termo de consentimento livre e esclarecido, a seguir devidamente assinado. Depois, os indivíduos preencheram a ficha de avaliação e anamnese, desenvolvidos pelo próprio autor.

Para avaliar o controle motor da região lombopélvica, os voluntários foram submetidos a três testes que envolvem movimentos ativos dos membros inferiores.

Os testes foram constituídos de movimentos ativos com os membros inferiores como: flexão de quadril, flexão de joelho, extensão de quadril e abdução/rotação lateral de quadril.

- O primeiro teste é denominado de Bent Knee fall out (BKFO): os voluntários foram posicionados em decúbito dorsal com um membro em extensão e o 
outro com flexão de joelho e quadril com o pé apoiado ao solo. O biofeedback de pressão foi colocado na coluna lombar, com a parte inferior na espinha ilíaca posterossuperior do lado contralateral ao membro em flexão. O joelho em flexão realizou abdução e rotação lateral do quadril de aproximadamente $45^{\circ}$ e retornou à posição inicial (ROUSSEL et al., 2009).

- O segundo teste é denominado de Knee lift abdominal test (KLAT), ou teste de flexão de quadril: consiste em posicionar os participantes em decúbito dorsal com flexão dos joelhos. Após essa etapa, o biofeedback de pressão foi colocado horizontalmente na coluna lombar dos voluntários com a parte inferior posicionada nas espinhas ilíacas posterossuperior. Para realizar o teste, os voluntários tiveram que realizar uma flexão de quadril retirando o pé do solo (HODGES, 2011). Essa flexão foi realizada em três fases: com $30^{\circ}$ de flexão de quadril, $60^{\circ}$ e $90^{\circ}$, que foram mensuradas com um goniômetro. A cada angulação foi verificada a variação de pressão. Se o indivíduo não conseguir chegar aos $90^{\circ}$ de flexão de quadril, é considerada a angulação máxima alcançada (AZEVEDO et al., 2013).

- O terceiro e último teste é denominado de Extensão de quadril: os participantes foram posicionados em decúbito ventral com os joelhos em extensão. O biofeedback de pressão foi posicionado na espinha ilíaca anterossuperior do lado homolateral ao membro a ser testado. Os voluntários foram instruídos a realizar a extensão de quadril de $15^{\circ}$ com extensão de joelho (AZEVEDO et al., 2013). Ao término de cada movimento, o avaliador registrou os valores referentes à pressão obtida pelo dispositivo de biofeedback, para posteriormente confrontar com o valor de base.

Após a conclusão da referida pesquisa, e de uma forma devolutiva, os autores se responsabilizam em apresentar os resultados e conclusão à população amostrada, bem como se comprometeram em orientar esses voluntários e/ou encaminhá-los para o ambulatório de fisioterapia Universidade Mogi da Cruzes, campus Villa-lobos-SP.

Com o objetivo de minimizar as possíveis intercorrencias quanto aos procedimentos a serem realizados no momento da coleta dos dados, e também, como forma de treinamento prévio ao avaliador, foi realizado um estudo piloto com uma amostra de dois indivíduos, que não foram incluídos na pesquisa. Este procedimento antecedeu a efetiva coleta de dados.

\section{Análise dos dados}

Os dados foram apresentados em tabela de contingência, e expressos por valores de frequência.

Para as medidas de significância foi utilizado o teste estatístico de $\mathrm{X}^{2}$ de independência $\left(\mathrm{x}^{2}=\right.$ qui-quadrado), com correção de continuidade de Yates, com nível de significância em 5\%.

Para as medidas de associação foi utilizado o teste estatístico do Coeficiente $\varphi$ (fi), segundo o qual quanto mais próximo estiver de 1 , maior o grau de associação entre as variáveis, aceitando valores maior ou igual a 0,30 . Valores menores que 0,30 foram considerados como de baixa associação entre as variáveis, ou associação nula quando igual a 0 (VIEIRA, 2003).

$\mathrm{H}_{0}$ (hipótese nula): Os sintomas de lombalgia independem do controle motor lombopélvico.

$\mathrm{H}_{1}$ (hipótese alternativa): Os sintomas de lombalgia dependem do controle motor da região lombopélvica.

Valor de $\mathrm{p}<0,05$, rejeita-se a hipótese nula e aceita a hipótese alternativa.

\section{Resultados}

Foram avaliados 80 lutadores de jiu-jitsu, com idade entre 18 a 42 anos, apresentando média de 28,9 anos. A média de peso corporal foi de $83,75 \mathrm{~kg}$, com altura média de $1,77 \mathrm{~m}$ e média de IMC (índice de massa corpórea) de $26,75 \mathrm{~kg} / \mathrm{m}^{2}$ (sobrepeso) (JONNALAGADDA et al., 2004) Tabela 1.

Os valores médios referentes à frequência dos treinos de jiu-jitsu foi de 3,28 vezes por semana, e a média do tempo de prática da luta foi de 3,8 anos.

Tabela 1 - Dados antropométricos dos lutadores de jiu-jitsu

\begin{tabular}{l|l}
\hline CARACTERÍSTICAS & MÉDIA \pm DP (MINIMO MÁXIMO) \\
\hline IDADE - anos & $28,9 \pm 6,15(18-42)$ \\
\hline PESO - kg & $83,75 \pm 14,34(61-120)$ \\
\hline ALTURA - m & $1,77 \pm 0,07(1,63-2,0)$ \\
\hline IMC - kg/m2 & $26,75 \pm 3,67(20,47-36,41)$ \\
\hline FREQUÊNCIA DE & $3,28 \pm 0,73(2-4)$ \\
TREINO - SEMANAL & \\
\hline TEMPO DE LUTA - ANOS & $3,8 \pm 4,40(0,9-20)$ \\
\hline
\end{tabular}


Entre os 80 voluntários, lutadores de jiu-jitsu, 24 deles informaram que praticam outro tipo de arte marcial, e 32 deles, correspondendo $40 \%$ da amostra, afirmaram ter dor lombar. A intensidade da dor foi aferida pela Escala Analógica de Dor - (0 a 10), com média de 5,65 , considerada como moderada. Tabela 2.

Em relação ao lado dominante, 7 lutadores relataram que seu lado dominante é o esquerdo; outros 7 relataram dominância de ambos os lados; e 66 afirmaram que o lado direito é o seu dominante.

Tabela 2 - Distribuição de sintomatologia de lombalgia e lado dominante do lutador

\begin{tabular}{l|c}
\hline CARACTERÍSTICAS & FREQUÊNCIA \\
\hline SINTOMAS DE LOMBALGIA & 32 \\
\hline PRATICANTE DE OUTRA ARTE MARCIAL & 24 \\
\hline LADO DOMINANTE ESQUERDO & 7 \\
\hline LADO DOMINANTE DIREITO & 66 \\
\hline AMBOS LADOS DOMINANTES & 7 \\
\hline
\end{tabular}

O teste Bent Knee fall out foi aplicado bilateralmente. Do lado direito apenas 13 indivíduos conseguiram realizar o teste com controle da região lombopélvica, sendo que apenas 3 desses voluntários tinham dor lombar; já 67 lutadores não conseguiram manter o controle motor durante o teste. $\mathrm{O}$ valor de $\mathrm{P}$ para esse teste é de $\mathrm{P}=0,2930$, não apresentando relação significativa com a sintomatologia de dor lombar e o défice do controle motor. Confirmando a independência do controle motor e a dor lombar, o valor do coeficiente $\varphi$ foi de 0,1522 .

Tabela 3 - Presença de controle motor em lutadores com e sem sintomatologia de dor lombar. Teste BKFO do lado direito

\begin{tabular}{c|c|c|c}
\hline \multicolumn{4}{|c}{ BENT KNEE FALL OUT (Direito) } \\
\hline $\begin{array}{c}\text { DOR } \\
\text { LOMBAR }\end{array}$ & CONTROLE MOTOR & $\begin{array}{c}\text { SEM CONTROLE } \\
\text { MOTOR }\end{array}$ & TOTAL \\
\hline SIM & 3 & 29 & 32 \\
\hline NÃO & 10 & 38 & 48 \\
\hline TOTAL & 13 & 67 & 80 \\
\hline
\end{tabular}

O mesmo teste do lado esquerdo apresentou apenas 16 lutadores que mantiveram o controle motor durante o teste, sendo que 6 desses lutadores relataram dor lombar. Assim como do lado direito, o valor de $\mathrm{P}=0,9545$ não apresentou significância estatística entre a dor lombar e controle motor nesses indivíduos. $O$ valor do coeficiente $\varphi$ também foi menor que 1 , apresentando valor de 0,0255 , confirmando a independência entre o défice do controle motor da região lombopélvica e a dor lombar.

Tabela 3.1 - Presença de controle motor em lutadores com e sem sintomatologia de dor lombar. Teste BKFO do lado esquerdo

\begin{tabular}{c|c|c|c}
\hline \multicolumn{4}{c}{ BENT KNEE FALL OUT (Esquerdo) } \\
\hline $\begin{array}{c}\text { DOR } \\
\text { LOMBAR }\end{array}$ & CONTROLE MOTOR & $\begin{array}{c}\text { SEM CONTROLE } \\
\text { MOTOR }\end{array}$ & TOTAL \\
\hline SIM & 6 & 26 & 32 \\
\hline NÃO & 10 & 38 & 48 \\
\hline TOTAL & 16 & 64 & 80 \\
\hline
\end{tabular}

No teste Knee lift abdominal test na angulação de $30^{\circ}$ do lado direito, 28 lutadores conseguiram manter o controle durante o teste, sendo que apenas 8 apresentaram sintomatologia de dor lombar. Já 52 lutadores não conseguiram manter o controle, dentre esses 24 apresentaram dor lombar. $\mathrm{O}$ valor de $\mathrm{P}=$ 0,1964 evidencia que não ocorre dependência entre a dor lombar e o déficit do controle motor. Confirmando a não associação, o valor do coeficiente $\varphi$ foi de 0,1712 .

Tabela 4 - Presença de controle motor em lutadores com e sem sintomatologia de dor lombar. Teste KLAT com angulação de $30^{\circ}$ de flexão de quadril do lado direito.

\begin{tabular}{c|c|c|c}
\hline \multicolumn{4}{c}{ KNEE LIFT ABDOMINAL TEST ( DIREITO ) } \\
$30^{\circ}$ \\
\hline $\begin{array}{c}\text { DOR } \\
\text { LOMBAR }\end{array}$ & CONTROLE MOTOR & $\begin{array}{c}\text { SEM CONTROLE } \\
\text { MOTOR }\end{array}$ & TOTAL \\
\hline SIM & 8 & 24 & 32 \\
\hline NÃO & 20 & 28 & 48 \\
\hline TOTAL & 28 & 52 & 80 \\
\hline
\end{tabular}


O mesmo teste foi realizado do lado esquerdo, sendo que 29 voluntários conseguiram manter o controle motor durante o teste, e 8 indivíduos apresentam dor lombar. A quantidade de lutadores que não apresentaram controle durante o teste foi de 51, e 24 desses lutadores apresentaram dor lombar. $\mathrm{O}$ valor de $\mathrm{P}$ para o lado esquerdo foi de 0,1411 , não apresentando significância da dependência da dor lombar e o défice do controle motor. $\mathrm{O}$ valor do coeficiente $\varphi$ também confirma essa não associação neste grupo, tendo valor de 0,1911 .

Tabela 4.1 - Presença de controle motor em lutadores com e sem sintomatologia de dor lombar. Teste KLAT com angulação de $30^{\circ}$ de flexão de quadril do lado esquerdo

\begin{tabular}{c|c|c|c}
\hline \multicolumn{4}{c}{$\begin{array}{c}\text { KNEE LIFT ABDOMINAL TEST (ESQUERDO ) } \\
30^{\circ}\end{array}$} \\
\hline $\begin{array}{c}\text { DOR } \\
\text { LOMBAR }\end{array}$ & CONTROLE MOTOR & $\begin{array}{c}\text { SEM CONTROLE } \\
\text { MOTOR }\end{array}$ & TOTAL \\
\hline SIM & 8 & 24 & 32 \\
\hline NÃO & 21 & 27 & 48 \\
\hline TOTAL & 29 & 51 & 80 \\
\hline
\end{tabular}

$\mathrm{O}$ teste Knee lift abdominal test com angulação de $60^{\circ}$ do lado direito apresentou apenas 25 voluntários que conseguiram manter o controle motor, sendo que 7 relataram sintomatologia de dor lombar, os restantes 55 lutadores não conseguiram manter o controle motor, dentre eles 25 apresentaram dor lombar. $\mathrm{O}$ valor de $\mathrm{P}$ deste teste foi de 0,2184 , mostrando que não há dependência entre a dor lombar e o défice do controle motor da região lombopélvica. Confirmando esse resultado, o coeficiente $\varphi$ foi de 0,1651, ou seja, confirmando a não associação entre essas variáveis.

Tabela 4.2 - Presença de controle motor em lutadores com e sem sintomatologia de dor lombar. Teste KLAT do lado direito com de $60^{\circ}$ de flexão de quadril

\begin{tabular}{c|c|c|c}
\hline \multicolumn{4}{c}{ KNEE LIFT ABDOMINAL TEST ( DIREITO ) } \\
$60^{\circ}$ \\
\hline $\begin{array}{c}\text { DOR } \\
\text { LOMBAR }\end{array}$ & CONTROLE MOTOR & $\begin{array}{c}\text { SEM CONTROLE } \\
\text { MOTOR }\end{array}$ & TOTAL \\
\hline SIM & 7 & 25 & 32 \\
\hline NÃO & 18 & 30 & 48 \\
\hline TOTAL & 25 & 55 & 80 \\
\hline
\end{tabular}

O Knee lift abdominal test com angulação de $60^{\circ}$ do lado esquerdo mostrou que 22 indivíduos conseguiram manter o controle motor durante o teste, e apenas 6 desses indivíduos apresentaram dor lombar, o total de lutadores que não conseguiram manter o controle motor foi de 58, sendo que 26 desse lutadores apresentam dor lombar. $\mathrm{O}$ valor de $\mathrm{P}$ para o lado esquerdo foi de 0,2398 , que mostra que não há dependência entre a dor lombar e o défice do controle motor; o valor do coeficiente $\varphi$ foi de 0,1600 , que confirma a não associação das variáveis, dor lombar e défice do controle motor.

Tabela 4.3 - Presença de controle motor em lutadores com e sem sintomatologia de dor lombar. Teste KLAT do lado esquerdo com $60^{\circ}$ de flexão de quadril

\begin{tabular}{c|c|c|c}
\hline \multicolumn{4}{c}{ KNEE LIFT ABDOMINAL TEST (ESQUERDO ) } \\
$60^{\circ}$ \\
\hline $\begin{array}{c}\text { DOR } \\
\text { LOMBAR }\end{array}$ & CONTROLE MOTOR & $\begin{array}{c}\text { SEM CONTROLE } \\
\text { MOTOR }\end{array}$ & TOTAL \\
\hline SIM & 6 & 26 & 32 \\
\hline NÃO & 16 & 32 & 48 \\
\hline TOTAL & 22 & 58 & 80 \\
\hline
\end{tabular}

A quantidade de indivíduos que conseguiram manter o controle motor durante o teste Knee lift abdominal com angulação de $90^{\circ}$ do lado direito foi de 12 lutadores, sendo que apenas 2 apresentaram dor lombar, e a quantidade de lutadores que não conseguiram manter o controle motor foi de 68 lutadores, entre eles 30 apresentam dor lombar. $\mathrm{O}$ valor de $\mathrm{P}$ deste teste foi de 0,1416 , apresentando que não há relação estatística entre o controle motor da região lombopélvica e a dor lombar; o valor do coeficiente $\varphi$ foi 0,2001 , que confirma que não há dependência entre o défice do controle motor e a sintomatologia de dor lombar.

Tabela 4.4 - Quantidade de lutadores que apresentam ou não a sintomatologia de dor lombar, e se mantiveram ou não o controle motor durante o teste KLAT do lado direito com $90^{\circ}$ de flexão de quadril

\begin{tabular}{c|c|c|c}
\hline \multicolumn{4}{c}{ KNEE LIFT ABDOMINAL TEST ( DIREITO ) } \\
$90^{\circ}$
\end{tabular}


O mesmo teste do lado esquerdo mostra que 14 voluntários apresentaram controle motor durante o teste, sendo que apenas 2 desses lutadores têm sintomatologia de dor lombar, já 66 lutadores não apresentaram controle durante o teste, sendo que 30 desses voluntários apresentam sintomatologia de dor lombar. $\mathrm{O} \mathrm{P}=0,0626$ mostra que não há dependência entre o controle motor da região lombopélvica e a dor lombar; e o valor do coeficiente $\varphi$ foi 0,2471 , confirmando a não dependência entre a sintomatologia de dor lombar e a falta do controle motor.

Tabela 4.5 - Presença de controle motor em lutadores com e sem sintomatologia de dor lombar. Teste KLAT do lado esquerdo com $90^{\circ}$ de flexão de quadril

\begin{tabular}{c|c|c|c}
\hline \multicolumn{4}{c}{ KNEE LIFT ABDOMINAL TEST (ESQUERDO ) } \\
$90^{\circ}$ & TOTAL \\
\hline $\begin{array}{c}\text { DOR } \\
\text { LOMBAR }\end{array}$ & CONTROLE MOTOR & $\begin{array}{c}\text { SEM CONTROLE } \\
\text { MOTOR }\end{array}$ & TOL \\
\hline SIM & 2 & 30 & 32 \\
\hline NÃO & 12 & 36 & 48 \\
\hline TOTAL & 14 & 66 & 80 \\
\hline
\end{tabular}

Durante o teste de Extensão do quadril direito, somente 6 lutadores conseguiram manter o controle motor da região lombopélvica, porém apenas 1 lutador apresentou o controle relatando dor lombar. Entretanto os voluntários que não conseguiram manter o controle somaram 74, entre eles 31 apresentam a sintomatologia de dor lombar. Sendo então o valor de $\mathrm{P}=0,4355$, também não apresentando dependência entre a dor lombar e o défice do controle motor. O coeficiente $\varphi$ foi igual a 0,1356 , confirmando que não há dependência entre a dor lombar e a falta do controle motor lombopélvico.

Tabela 5 - Presença de controle motor em lutadores com e sem sintomatologia de dor lombar. Teste de Extensão de quadril do lado direito

\begin{tabular}{c|c|c|c}
\hline \multicolumn{4}{|c}{ EXTENSÃO DE QUADRIL (DIREITO) } \\
\hline $\begin{array}{c}\text { DOR } \\
\text { LOMBAR }\end{array}$ & CONTROLE MOTOR & $\begin{array}{c}\text { SEM CONTROLE } \\
\text { MOTOR }\end{array}$ & TOTAL \\
\hline SIM & 1 & 31 & 32 \\
\hline NÃO & 5 & 43 & 48 \\
\hline TOTAL & 6 & 74 & 80 \\
\hline
\end{tabular}

O teste de Extensão do quadril também foi realizado do lado esquerdo, em que 8 voluntários conseguiram realizar o teste e manter o controle motor, entre eles apenas 2 relataram dor lombar. Enquanto os voluntários que não conseguiram realizar o teste com o controle motor lombopélvico somaram 72, composto por 30 lutadores com dor lombar. O valor de $\mathrm{P}$ do lado esquerdo foi 0,5944 , mostrando que nessa amostra não houve dependência entre a dor lombar e a falta de controle motor da região lombopélvica; e o coeficiente $\varphi$ foi igual a 0,1021 , confirmando que não há dependência entre a dor lombar e o défice do controle motor da região lombopélvica.

Tabela 5.1 - Presença de controle motor em lutadores com e sem sintomatologia de dor lombar. Teste de Extensão de quadril do lado esquerdo

EXTENSÃO DE QUADRIL (ESQUERDO)

\begin{tabular}{c|c|c|c}
\hline $\begin{array}{c}\text { DOR } \\
\text { LOMBAR }\end{array}$ & CONTROLE MOTOR & $\begin{array}{c}\text { SEM CONTROLE } \\
\text { MOTOR }\end{array}$ & TOTAL \\
\hline SIM & 2 & 30 & 32 \\
\hline NÃO & 6 & 42 & 48 \\
\hline TOTAL & 8 & 72 & 80 \\
\hline
\end{tabular}

\section{Discussão}

Os episódios álgicos da região lombar da coluna vertebral é um evento doloroso que pode estar presente em atletas e praticantes de diferentes modalidades esportivas e/ou recreativas. A lombalgia é considerada como uma das responsáveis pela redução do rendimento esportivo, podendo ser a causa pelo insucesso numa competição, impedindo o atleta de alcançar êxito em sua prática (BONO, 2004; IWAI et al., 2004).

A dor lombar é um dos sintomas mais comuns na sociedade, em atletas jovens a prevalência de lombalgia é de 10 a $15 \%$, independente da modalidade ou do esporte praticado, mostrando uma maior prevalência em ginastas, com aproximadamente $86 \%$ com sintomas de lombalgia (PURCELL et al., 2009).

Outro esporte em que a lombalgia é muito comum é o golfe, com $52 \%$ de prevalência de dor lombar em praticantes norte-americanos. $\mathrm{O}$ gasto público ultrapassa o montante de U\$ 50 bilhões a cada ano com tratamento e prevenção da dor lombar associada à prática de golfe (GLUCK et al., 2008). 
Em estudos de prevalência de lombalgia em atletas de luta olímpica do Brasil e Estados Unidos da América, pode-se verificar uma prevalência de 59\% a $69 \%$ dos relatos álgicos respectivamente, além de associá-los às alterações posturais (DEZAN et al., 2004; BONO, 2004).

A presente pesquisa revelou uma prevalência de $40 \%$ de lombalgia em lutadores de jiu-jitsu, porém, não foi objeto de estudo a associação com as possíveis alterações posturais conforme o estudo anterior.

Em decorrência da escassez de literatura referente à prática de jiu-jitsu, não foi possível correlacioná-la com outras pesquisas.

Contudo, o objetivo da atual pesquisa foi analisar o controle motor dos músculos lombopélvicos e a presença de lombalgia em praticantes de jiu-jitsu. As variáveis não apresentaram associação pelos testes estatísticos.

Em um estudo comparativo realizado na Bélgica, os autores avaliaram a prevalência de dor lombar e o défice do controle motor lombopélvico em dançarinas; a amostra foi composta por 39 dançarinas, das quais 16 , ou seja, $41 \%$ da amostra, relataram dor lombar. Nesse mesmo estudo a comparação do controle motor foi através do biofeedback de pressão utilizando os testes KLAT e o BKFO, onde o grupo sem sintomatologia de dor lombar manteve as pressões durante os testes mais próximas aos 40 $\mathrm{mmHg}$, demonstrando-se que o grupo com lombalgia tem um défice maior do controle motor da região lombopélvica (ROUSSEL et al., 2013).

Sugere-se que falta do controle motor seja uma alteração do planejamento motor, ou melhor, os músculos estabilizadores da coluna lombar devem contrair-se antecipadamente à movimentação dos membros, assim estabilizando-a. Sabe-se também que indivíduos com dor lombar recorrente apresentam mudança no córtex motor que representa o músculo transverso do abdome, o que pode contribuir com o défice do controle postural e das respostas antecipatórias, além do músculo transverso do abdome, os músculos multifídios lombares também apresentaram défice nas respostas antecipatórias (HODGES et al., 2003; TSAO et al., 2008).

Indivíduos com dor lombar não apresentam a capacidade de antecipar a contração dos músculos estabilizadores durante a movimentação dos mem- bros, o que pode deixar a coluna lombar desprotegida das forças aplicadas e resultante dos movimentos dos membros inferiores (HODGES et al.,1998).

Em um estudo de intervenção com 30 indivíduos com dor lombar, divididos em dois grupos: grupo tratado com exercícios de estabilização segmentar, e grupo tratamento com fortalecimento dos músculos superficiais (músculo reto do abdome, músculo oblíquo interno e externo, e músculos eretores da espinha). Após o treinamento com duração de seis semanas, o grupo 'estabilização segmentar' apresentou redução da dor, além de uma melhor capacidade de ativação do músculo transverso do abdome; ao contrário do outro grupo, que apresentou uma menor capacidade de ativação muscular (FRANÇA et al., 2010).

O mesmo princípio pode ter ocorrido com os lutadores do presente estudo, já que o gesto esportivo da luta de jiu-jitsu induz o fortalecimento dos músculos superficiais, promovendo o défice do controle motor da musculatura profunda.

Visto que a falta de controle motor da região lombopélvica é uma condição que propicia o desenvolvimento de lesões, seria interessante a implantação de um programa de exercícios específicos denominados de Estabilização de Core, que visa à estabilização principalmente da região lombopélvica, fornecendo uma base para a movimentação dos membros superiores e inferiores, como também um suporte para cargas impostas à coluna vertebral (WILLARDSON, 2007).

O treinamento de Core é muito utilizado para a reabilitação de dor lombar, apresentando efetividade da redução da dor e aumento do recrutamento dos músculos estabilizadores (WILLARDSON, 2007; AKUTHOTA et al., 2004; BORGHUIS et al., 2008).

Os resultados da presente pesquisa foram favoráveis à aceitação da hipótese de nulidade, em que o défice do controle motor da região lombopélvica torna-se independente da sintomatologia de dor lombar em praticantes da luta marcial jiu-jitsu.

A prática da luta de jiu-jitsu, como uma arte marcial, vem em constante ascendência, com isso, destaque-se a necessidade e importância de pesquisas que correlacionem a prevalência de dor lombar e a ativação dos músculos estabilizadores, o que poderá responder sobre o défice do controle motor encontrado nestes praticantes. 


\section{Conclusão}

A população amostral analisada neste estudo não apresentou controle motor da musculatura lombopélvica pelo método de avaliação de biofeedback de pressão.

Os testes estatísticos não apresentaram associação entre a instabilidade lombopélvica e a sintomatologia de dor lombar.

\section{Referências}

AKUTHOTA, V; NADLER, S. F. Core strengthening. Arch Phys Med Rehabil, v.85, Suppl 1, March 2004.

AZEVEDO, D. C; LAURIA, A. C; PEREIRA, A. R. S; ANDRADE, G. T; FERREIRA, M. L; FERREIRA, P. H; DILEN, L. V. Intraexaminer and Interexaminer reliability of pressure biofeedback unit for assessing lumbopelvic stability during 6 lower limb movement tests. Journal of manipulative and physiological therapeutics, v.36, i.1, p.33-43, January, 2013.

BARBOSA, F. S. S ; GONÇALVES, M. Comparação entre protocolos de exaustão e de 30 segundos utilizados na avaliação da fadiga eletromiográfica dos músculos eretores da espinha. Rev. Bras. Fisioter. v.9, n.1. p. 77-83, 2005.

BONO, C. M. Low back pain in athletes. J. Bone Joint Surg. Am., v. 86, p. 382-396, 2004.

BORGHUIS, J; HOF, A. L; LEMMINK, K. A. P. M. The importance of sensory-motor control in providing core stability. Sport Med. v. 38, n. 1, p. 893-916, 2008.

CONFEDERAÇÃO Brasileira de Jiu-jitsu. Disponível em: $<$ http://www.cbjj.com.br/hjj.htm>.

DEZAN, V. H; SARRAF, T. A; RODACKI, A. L. F. Alterações posturais, desequilíbrios musculares e lombalgias em atletas de luta olímpica. R. Brás. Ci e Mov., v. 12, n. 1, p. 35-38, 2004.

FRANÇA, F. R; BURKE, T. N; HANADA, E. S; MARQUES, A. P. Segmental stabilization and muscular strengthening in chronic low back pain: a comparative study. Clinics; v. 65, n. 10, p. 1013-1017, 2010.

GLUCK, G. S; BENDO, J. A; SPIVAK, J. M. The lumbar spine and low back pain in golf: a literature review of swing biomechanics and injury prevention. The Spine Journal., v. 8, p. 778-788, 2008.

GURGE, L. F. Personal Jiu-jitsu. Rio de Janeiro: Axcel Books, 2003.

HERRINGTON, L; DAVIES, R. The influence of pilates training on the ability to contract the Transversus abdominis muscle in asymptomatic individuals. Journal of bodywork and movement therapies., v. 9, p. 52-57, 2005.
HODGES, P. W. Pain and motor control: from the laboratory to rehabilitation. Journal of Electromyography and Kinesiology., v. 21, p.220-228, 2011.

.; MOSELEY, G. L. Pain and motor contor of the lumbopelvic region: effect and possible mechanisms. Journal of Electromyography and Kinesiology. v.13 p. 361-370, 2003.

HODGES, P. W; RICHARDSON, C. Delayed postural contraction of transverses abdominis in low back pain associated with movement of lower limb. J Spinal Disord; v. 11, n. 1, p. 46-56, Feb 1998.

IWAI, K; NAKAZATO, K; IRIE, K; FUJIMOTO, H; NAKAJIMA, H. Trunk Muscle Strength and disability level of low back pain in collegiate wrestlers. Med. Sci. Sports Exerc., v.36, n. 8, p.1296-1300, 2004.

JONNALAGADDA, S. S; SKINNER, R; MOORE, L. Overweight athlete: fact or fiction? Current Sport Medicine Reports., v. 3, p. 198-205, 2004.

PANJABI, M. M. The stabilizing system of the spine, part 1: function, dysfunction, adaption and enhancement. J Spinal Disord., v.5, p. 383-9, 1992.

PHROMPAET, S; PAUNGMALI, A; PIRUNSAN, U; SITILERTPISAN, P. Effects of Pilates training on lumbopelvic stability and flexibility. Asian Journal of sports medicine, v.2, n.1, p. 16-22, 2011.

PURCELL, L; MICHELI, L. Low back pain in young athletes. Sports Health, May, v.1, n.3 2009.

ROUSSEL, N; KOONING, M; SCHUTT, A; MOTTRAM, S; TRUIJEN, S; DAENEN, L. Motor control and low back pain in dancers. Journal Sports Med., v.34, p. 138-143, 2013.

ROUSSEL, N; NIJS, J; TRUIJEN, S; VERVECKEN, L; MOTTRAM, S; STASSIJNS, G. Altered breathing patterns during lumbopelvic motor control tests in chronic low back pain: a case-control study. J Eur Spine., v.18, p. 1066-1073, 2009.

SOUZA, J. M. C; FAIM, F. T; NAKASHIMA, I. Y; ALTRUDA, C. R; MEDEIROS, W. M; SILVA, L. R. Lesões no karate shotokan e no jiu-jitsu: trauma direto versus indireto. Rev Brasileira de Medicina do Esporte. v. 17, n.2, Mar/ Abr, 2011.

TSAO, H.; GALEA, M. P; HODGES, P. W. Reorganization of the motor cortex is associated with postural control deficits in recurrent low back pain. Brain., v.131, p.21622171, 2008.

VECCHIO, F. B. D; BIANCHI, S.; HIRATA, S. M.; CHACON-MIKAHIL, M. P. T. Análise morfo-funcional de praticantes de Brasilian jiu-jitsu e estudo da temporalidade e da qualificação das ações motoras na modalidade. Movimento \& Percepção, Espírito Santo do Pinhal, SP., v.7, n.10, jan./jun.2007. 
VIEIRA, S. Bioestatística: tópicos avançados. 2.ed. São Paulo: Campus/ Elsevier, 2003. p. 63-63.

WILLARDSON, J. M. Core stability training: applications to sports conditioning programs. Journal of Strength and conditioning research, v. 21, n. 3, p. 979-985, 2007. 